\title{
Where Should Critically Ill Neurologic Brain Hemorrhage Patients Go and Can Transfer Harm Them?
}

\author{
Paul Nyquist
}

Published online: 11 September 2012

(C) Springer Science+Business Media, LLC 2012

\section{Introduction}

The brain is a complex organ subjected to acute neurological emergencies requiring rapid specialized care. With the advent of rTPA for acute ischemic stroke, the concept of aggressive intervention in neurological emergencies has grown to encompass other types of neurological emergencies such as intracranial hemorrhage and traumatic brain injury. This change has been fueled by the emergence of specialists who run and work in specialty units like the Neurocritical care unit (NCCU). These units have been shown to reduce morbidity, mortality, and the length of stay for critically ill neurologic patients [1-3]. The improved care in these units is driven by two underlying factors. The availability of technological interventions that are performed in the context of neurological specialty units and the concentration of neurological patients in a single location were the judgment and experience of specialty providers results in better outcomes [1-3].

Brain hemorrhage of all types, including intracranial (ICH) and aneurysmal subarachnoid (aSAH), is a subset of acute neurological emergencies for which truly definitive neurological care is now done in the setting of the NCCU. As we see the convergence of patients suffering from brain hemorrhages into the NCCU, issues arise pertaining to the need to transfer these patients to the NCCU in a rapid manner. What is the effect of urgent treatment in the NCCU on improved outcome for these neurological

\footnotetext{
P. Nyquist $(\square)$

Departments of Neurology, Anesthesiology/Critical Care

Medicine, and Neurosurgery, Johns Hopkins School of

Medicine, Meyer 8-140, 600 N Wolfe St., Baltimore,

MD 21287, USA

e-mail: Pnyquis1@jhmi.edu
}

emergencies? Does the risk of transfer, or delay of transfer, affect outcomes? Three articles published in Neurocritical care present findings which raise questions about the effects of transfer and delay of transfer on outcomes in patients with ICH and aSAH.

\section{Delay of Transfer from the Emergency Department}

Extended, emergency department length of stay (EDLOS) is a variable affecting many medical conditions and has been associated with worse medical outcomes in acutely ill medical and neurological patients [4]. As EDLOS is increased, due to hospital congestion or other obstacles, critically ill patients are cared for in the emergency department (ED) often with worse outcomes. Recently, Elmer et al. [5] published a study in Neurocritical Care, documenting 574 prospective ICH emergency room encounters. In this study, a wide number of prospectively obtained variables including disease severity, IVH placement, and ventilator status were measured and compared to markers of morbidity. These included the modified Rankin scale (mRS), and secondary outcomes such as ICU length of stay, total hospital length of stay, and total hospital costs. This study was well conducted, with good study design, using prospectively gathered data. It had very detailed and involved analysis of all variables. No harm related to EDLOS up to $7 \mathrm{~h}$ was identified in this study. Based on this study, it appears that ICH patients may not be significantly affected by increased EDLOS. This is an interesting finding and quite contrary to what we would have expected based on EDLOS and its relation to other medical illness. Despite its strengths, this study was completed in a high-level tertiary care center in which neurosurgical and neurological support were readily 
available in the ED. These resources are not available in the standard community hospital and the issues of transfer and delay have not truly been investigated in that setting. At present, our understanding of ICH is incomplete and we need to do a definitive community study to see how EDLOS affects outcomes in ICH.

\section{Harm from Transfer}

The effectiveness and utility of transfer revolves around the risk benefit analysis of patient movement versus the benefit of specialized care. Almost all patients who are acutely ill suffer harm in the process of inter-hospital transfer. The increased risk and associated morbidity of inter-hospital transfer has been studied in the general medical population [6-8]. Transferred patients tend to be sicker and have higher overall mortality, and often suffer additional morbidity from transfer [6-8]. Most often the harm of transfer is outweighed by the benefits of specialized care. For instance with myocardial infarction and acute trauma, both medical and surgical interventions positively benefit patients in a manner that outweighs the risk of transfer and improves outcomes [9-11].

Two articles published in this issue of Neurocritical care address issues pertaining to the morbidity and mortality associated with the inter-hospital transfer of aSAH. Catalano et al. has prospectively studied mortality and morbidity in inter-hospital transfer in aSAH. While the study was small with $120(47 \%)$ of patient transfers observed from a total of 247 aSAH ED admissions, and limited to a single center, some important observations were made. The mortality of all patients transferred did not vary based on delay of transfer. However, significant increased risk of aneurysmal rebleed ( 7.3 vs. $1.8 \%$, $P=0.007)$ as well as an increasing risk of tracheostomy were observed in the transfer group (20 vs. $17.5 \%$, $\mathrm{P}=0.013$ ) [12]. Cognitive outcomes were reportedly worse in transferred patients as compared to direct admissions. This study would seem to suggest that there is significant harm associated with the transfer of aSAH patients to tertiary care centers. Nuño et al. published an article in this issue, addressing similar questions in a retrospective analysis of a large national database. The effects of transfer on outcomes were analyzed from the Nationwide Inpatient Sample (NIS) 2002-2007 data. This included 47,114 patients, 31,711 (67.3\%) were direct admits, and 15,403 (32.7\%) were transfers [13]. Variables included, age, disease severity, need for ventriculostomy, and type of procedure. There were no differences related to increased mortality and morbidity secondary to transfer or delay of transfer. However, individuals treated in low volume centers were 1.6 times as likely to die as those treated at high volume centers.

\section{Conclusions}

In general, available data support the idea that neurological patients receive benefits from care in the specialty NCCU's. Presently, studies on patients with ICH and aSAH suggest that the benefit of transfer and the cost of delay may not be what we suspect. Specifically, increasing EDLOS may not be detrimental to outcomes if minimal adequate neurological care can be obtained in the ED. Patient transport may be more harmful to patients with aSAH than suspected with increasing risk of aneurysmal rupture and possible worse cognitive outcomes. The care received at tertiary centers specializing in aSAH care seems to be beneficial. These studies lay the ground work for future studies that must address the cost and benefits of transfer of these patients to specialty NCCUs. These future studies must integrate all aspect of care into single model to answer the important questions surrounding his issue. At this time, the definitive study to clearly define the risk and benefits of referral of brain hemorrhage patients has not been done.

How do we design better future studies based on the present experience. Retrospective studies offer the chance to look at broad questions of outcome with greater power. In general, they do not grant insight into the decisions that drive patient outcomes. The Nuño et al. study allows us to look at mortality from aSAH TX over an extended period of time measured in months to years. It cannot tell us about the decisions made by ED doctors over minutes to hours. This type of database study suffers from recall bias and bias from the use of data tabulated for other purposes. The observation that aSAH do better at specialty centers is probably a valid conclusion. However, the data relating to transfer and the timing of transfer is dubious and it is unlikely that harm from transfer can be studied in the context of such a design. The prospective study by Catalano et al. observed associations between decreasing cognitive outcomes and increased tracheostomy placement with inter-hospital transfer. While being truly prospective, the power of this study is limited by 120 observations and small numbers of observed adverse events. It lacks the power to analyze details leading to these bad outcomes. In addition, the study does not provide any insight or information about medical decision making leading to transfer.

To construct a study that allows for a true understanding of how transfer to specialty NCCU affects outcome, we must address design challenges unique to the transfer of critically ill patients. Critically ill patients are medically complex and any study would need to be large to enough to 
power the analysis of many complex variables. The study would need to incorporate a decision analysis of physician's decisions prior to and after transfer, and relate these decisions to changes in the patient's condition over time. It would need to be population based, and must ensure that the patients are treated in a similar manner regardless of which unit they are cared in. This would require that all patients are captured prospectively and observed in a large number of ED's prior to transfer to a small number of referral centers and their specialty units. Finally, it is likely that a number of specialized instruments must be developed and validated to address disease severity and outcomes incurred during transfer.

The Neurocritical care community must act to study this issue with the goal of improving patient outcomes. Furthermore, this study must be the beginning of a larger endeavor to continually update and critique our models of care. To do this, we need to use our existing research networks to develop and fund a study of sufficient power and complexity to support investigation of this issue on an ongoing basis. Sources of funding are scarce as the present financial crisis affects NIH dollars. There are a number of alternative funding sources including, the Gates foundation, AHA, and Medicaid and Medicare through CMS. We must exploit these smaller catchments of funding to help support a larger goal of understanding how our system of care affects patient outcomes in the community.

\section{References}

1. Diringer MN, Edwards DF. Admission to a neurologic/neurosurgical intensive care unit is associated with reduced mortality rate after intracerebral hemorrhage. Crit Care Med. 2001;29:635-40.

2. Mirski MA, Chang CW, Cowan R. Impact of a neuroscience intensive care unit on neurosurgical patient outcomes and cost of care: evidence-based support for an intensivist-directed specialty ICU model of care. J Neurosurg Anesthesiol. 2001;13(2):83-92.
3. Suarez JI, Zaidat OO, Suri MF, Feen ES, Lynch G, Hickman J, et al. Length of stay and mortality in neurocritically ill patients: impact of a specialized neurocritical care team. Crit Care Med. 2004;32(11):2311-7.

4. Chalfin DB, Trzeciak S, Likourezos A, Baumann BM, Dellinger RP. Impact of delayed transfer of critically ill patients from the emergency department to the intensive care unit. Crit Care Med. 2007;35:1477-83.

5. Elmer J, Pallin DJ, Shan L, Pearson C, Chang Y et al. Prolonged emergency department length of stay is not associated with worse outcomes in patients with intracerebral hemorrhage. Neurocrit Care. 2011. doi:10.1007/s12028-011-9629-1.

6. Schiff RL, Ansell DA, Schlosser JE, Idris AH, Morrison A, Whitman S. Transfers to a public hospital. A prospective study of 467 patients. N Engl J Med. 1986;314:552-7.

7. Ligtenberg JJ, Arnold LG, Stienstra Y, et al. Quality of interhospital transport of critically ill patients: a prospective audit. Crit Care. 2005;9:R446-51.

8. Durairaj L, Will JG, Torner JC, Doebbeling BN. Prognostic factors for mortality following interhospital transfers to the medical intensive care unit of a tertiary referral center. Crit Care Med. 2003;31:1981-6.

9. Sampalis JS, Denis R, Frechette P, Brown R, Fleiszer D, Mulder $D$. Direct transport to tertiary trauma centers versus transfer from lower level facilities: impact on mortality and morbidity among patients with major trauma. J Trauma. 1997;43(2):288-95.

10. Westfall JM, Kiefe CI, Weissman NW, et al. Does interhospital transfer improve outcome of acute myocardial infarction? A propensity score analysis from the Cardiovascular Cooperative Project. BMC Cardiovasc Disord. 2008;8:22.

11. Antman EM, Anbe DT, Armstrong PW, Bates ER, et al. ACC/ AHA guidelines for the management of patients with ST-elevation myocardial infarction; A report of the American College of Cardiology/American Heart Association Task Force on Practice Guidelines (Committee to Revise the 1999 Guidelines for the Management of patients with acute myocardial infarction). J Am Coll Cardiol. 2004;44:E1-211.

12. Catalano R, Winn HR, Gordon E, Frontera JA. Impact of interhospital transfer on complications and outcome after intracranial hemorrhage. Neurocrit Care. 2012. doi:10.1007/s12028-0129679-z.

13. Nuno M, Patil CG, Lyden P, Drazin D, The effect of transfer and hospital volume in subarachnoid hemorrhage patients. Neurocritical care. 2012. doi:10.1007/s12028-012-9740-y. 